\title{
Clinical situations in which coronary CT angiography confers superior diagnostic information compared with coronary angiography
}

\author{
Mecit Kantarcı, Selim Doğanay, Muşturay Karçaaltıncaba, Nevzat Karabulut, Mustafa Kemal Erol, \\ Ahmet Yalçın, Cihan Duran, Memduh Dursun, Afak Karakaya, Servet Tatlı
}

\begin{abstract}
In this review, we aimed to outline the clinical and pathological conditions for which multidetector computed tomography coronary angiography (MDCT-CA) should be the preferred method because of its advantages over conventional coronary angiography (CCA). A consistent body of literature suggests that MDCT-CA is more than just complementary to CCA and that it provides more valuable diagnostic information in certain clinical situations, such as complex coronary artery variations, aorto-ostial lesions, follow-up of bypass grafts, myocardial bridging, coronary artery fistulas, aortic and coronary artery dissections, and cases in which the coronary ostia cannot be cannulated by a catheter because of massive atherosclerosis or extremely tortuous vascular structures.

Key words: • computed tomography • coronary angiography - coronary arteries
\end{abstract}

From the Department of Radiology (M.KantarcI, A.Y. $\square$ dr_ayalcin@hotmail.com, A.K.), Atatürk University School of Medicine, Erzurum, Turkey; the Department of Radiology (S.D.), Erciyes University School of Medicine, Kayseri, Turkey; the Department of Radiology (M.Karçaaltıncaba), Hacettepe University School of Medicine, Ankara, Turkey; the Department of Radiology (N.K.), Pamukkale University School of Medicine, Denizli, Turkey; the Department of Cardiology (M.K.E.), Atatürk University School of Medicine, Erzurum, Turkey; the Department of Radiology (C.D.), Bilim University School of Medicine, İstanbul, Turkey; the Department of Radiology (M.D.), İstanbul University İstanbul School of Medicine, İstanbul, Turkey; the Department of Radiology (S.T.), Harvard Medical School, Brigham and Women's Hospital, Boston, Massachusetts, USA.

Received 4 October 2011; accepted 5 October 2011.

Published online 19 January 2012

DOI 10.4261/1305-3825.DIR.5064-11.1

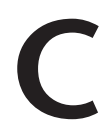
oronary artery disease is responsible for millions of deaths per year in developed countries $(1,2)$. Early recognition of coronary artery disease is important for the prevention of its related complications and improving prognosis (3). Catheter angiography has been considered the gold standard imaging method for evaluating the coronary tree. The major advantages of conventional coronary angiography (CCA) are its high spatial and temporal resolutions and the option of directly performing interventions, such as balloon dilation or coronary stent placement (4). However, CCA is an invasive procedure that carries the risk of morbidity and mortality (3). In addition, CCA provides only luminal data and is limited in revealing changes in the vessel wall and the adjacent soft tissues. Conversely, multidetector computed tomography coronary angiography (MDCT-CA) not only provides visualization of the vessel lumen but also displays the vessel wall and surrounding soft tissues, allowing for the detection of various congenital or acquired abnormalities of the coronary arteries that may not be evident with CCA (3).

In this article, on the basis of our experience with more than 15000 MDCT-CA from seven different centers, we reviewed clinical conditions in which MDCT-CA may be more helpful compared with CCA.

\section{Coronary CT technique}

With the advent of technological refinements in the last decade, MDCT-CA has gained widespread use for the evaluation of coronary artery diseases. Currently, state-of-the-art computed tomography (CT) systems can simultaneously acquire 64 or more submillimeter sections with a gantry rotation time of less than half a second. Previous limitations of CT for evaluating the vascular system, associated with older generation scanners, have now been eliminated. Presently, a thinner section thickness allows for isotropic voxels, which is essential for optimum high-resolution, three-dimensional (3D) reconstruction and other postprocessing displays, such as maximum projection reformatting (MPR) in any selected plane. Faster scanners have resulted in shorter scans and shorter contrast bolus durations, allowing for the use of less contrast agent at a higher flow rate to achieve greater luminal enhancement. Motion artifacts from breathing are no longer a problem since highresolution imaging of the entire heart can be obtained in a single breath hold. Electrocardiography (ECG) gating techniques improve temporal resolution and minimize imaging artifacts caused by cardiac motion. With more recent technological advances, a reduction in radiation dose, for example, by the utilization of prospective gating, has occurred without any deterioration in diagnostic image quality (5).

In our centers, images were acquired using either 16- or 64-MDCT scanners. Eighty-five to one hundred milliliters of iodinated contrast medium (Ultravist 370, Schering, Berlin, Germany) was intravenously 

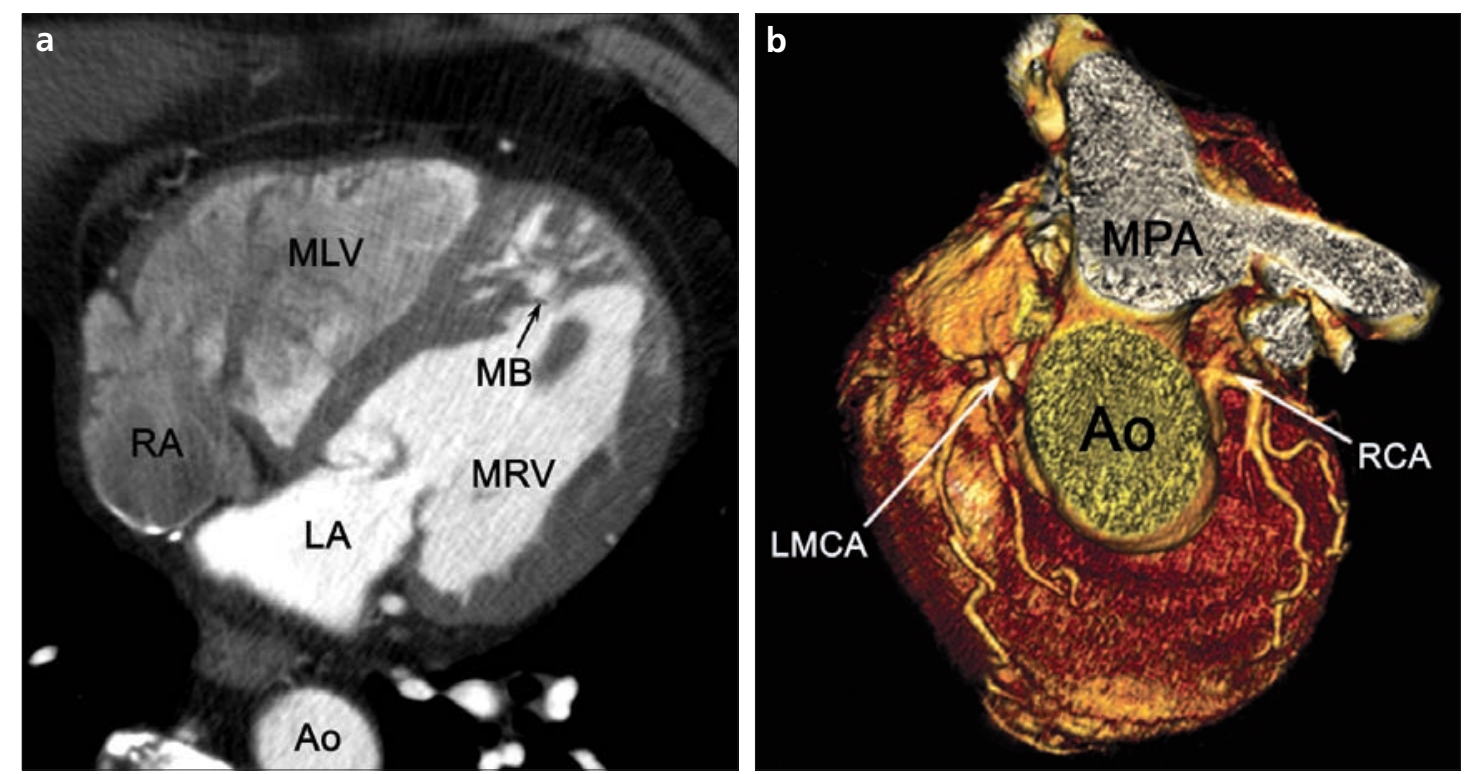

Figure 1. a, b. A congenitally corrected transposition of the great arteries in a 56-year-old man. The axial MIP image (a) shows that the right atrium (RA) connects to the morphologic left ventricle (MLV) located on the right. Note the characteristic moderator band (MB, arrow) within the morphologic right ventricle (MRV) on the left. An anterior view of a 3D volume-rendered image (b), at the level of the origin of the great arteries, shows the topographic relationship of the vessels. The ascending aorta (Ao) is anterior to and left of the main pulmonary artery (MPA) (b). LA, left atrium; LMCA, left main coronary artery; RCA, right coronary artery.

injected at $4.5 \mathrm{~mL} / \mathrm{s}$ followed by 40 $\mathrm{mL}$ of saline at $2.5 \mathrm{~mL} / \mathrm{s}$. Retrospective ECG-gated reconstructions were generated at $50 \%, 60 \%$, and $70 \%$ of the R-R interval of the ECG cycle. The maximum intensity projection (MIP) images and curved multiplanar MPR of the coronary arteries were routinely acquired. The patients were premedicated with nitroglycerin (5 mg sublingual administration $1 \mathrm{~min}$ before the examination to dilate the coronary arteries) and with metoprolol $(5 \mathrm{mg} / \mathrm{mL}$ bolus delivered intravenously to decrease the heart rate).

\section{Clinical situations in which MDCT- CA provides superior diagnostic information compared with CCA}

By simultaneously displaying the heart, great vessels, and coronary arteries, MDCT-CA readily evaluates complex coronary artery variations, aortoostial lesions, bypass grafts, myocardial bridging, coronary artery fistulas, and aortic and coronary artery dissections. This approach is also superior to CCA for cases in which the coronary ostia cannot be cannulated by a catheter because of massive atherosclerosis or extremely tortuous vascular structures.

\section{Complex coronary artery variations}

MDCT-CA is more successful in imaging coronary artery variations than
CCA because of its 3D volume-rendering ability (6). The most striking variations are listed below.

\section{Congenitally corrected transposition of the great arteries}

Congenitally corrected transposition of the great arteries (CCTGA) is a rare cardiac anomaly characterized by atrioventricular (AV) and ventriculoarterial (VA) discordance $(7,8)$. In patients with CCTGA, the atrial situs relationship is normal; the right atrium (RA) is to the right of the left atrium (LA). The RA empties into the ventricle located on the right (morphologically, the left ventricle [LV]) through the mitral valve, and the main pulmonary artery originates from this ventricle. This chamber has papillary muscles and a smooth septal wall. The LA empties into the ventricle located on the left (morphologically, the right ventricle $[\mathrm{RV}])$ through the tricuspid valve, and the aorta arises from this ventricle. This ventricle has prominent trabeculations and a moderator band (Fig. 1a). The aorta is located left of and anterior to the pulmonary artery (Fig. 1b) $(7,9)$. In patients with CCTGA, the coronary artery anatomy is more complex, and the coronary arteries show a mirror-image distribution. The morphologic right coronary artery (RCA) arises from the left posterior sinus, and the morphologic left main coronary artery (LMCA) arises from the right anterior sinus. The RCA supplies the anterior descending branch and gives rise to a circumflex (Cx) artery. In addition, the LMCA resembles an RCA (7).

MDCT-CA is able to demonstrate the morphology of the heart (ventricles, interventricular septum, and walls of the heart) and coronary arteries as well as the location of the large vessels originating from the heart by using 3D volume rendering and MIP images obtained in three planes (7).

\section{Agenesis of the LMCA}

The LMCA is absent in $0.41 \%$ of cases (6). In this condition, the left anterior descending artery (LADA) and $\mathrm{Cx}$ artery arise separately or with a common ostium. This anomaly is common and accounts for $30.4 \%$ of all coronary anomalies $(6,10)$. If the LMCA is absent, one of the following results may occur: a) the LADA and left $\mathrm{Cx}$ arteries, both of normal length and course, may arise from the left sinus of Valsalva separately or with a common ostium (Fig. 2) (11, 12); b) the left Cx artery may arise from the right sinus of Valsalva or the RCA and follow a course posterior to the aorta (13-15); c) the LADA may arise from the right sinus of Valsalva or the RCA (14-16) 


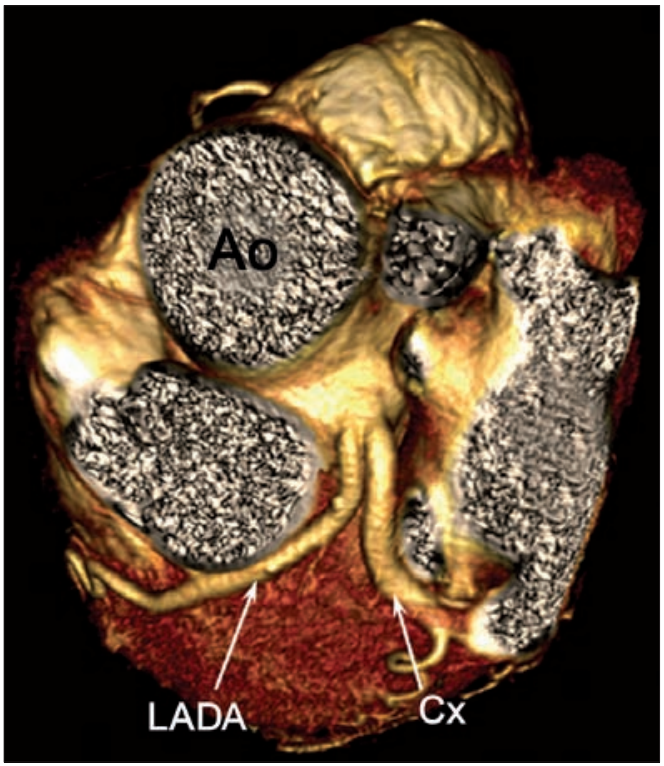

Figure 2. Agenesis of the left main coronary artery (LMCA) in a 64-year-old woman. An anterosuperior view of a 3D volume-rendered image shows the left anterior descending artery (LADA) and circumflex (Cx) artery arising separately from the left sinus of Valsalva. Ao, aorta.

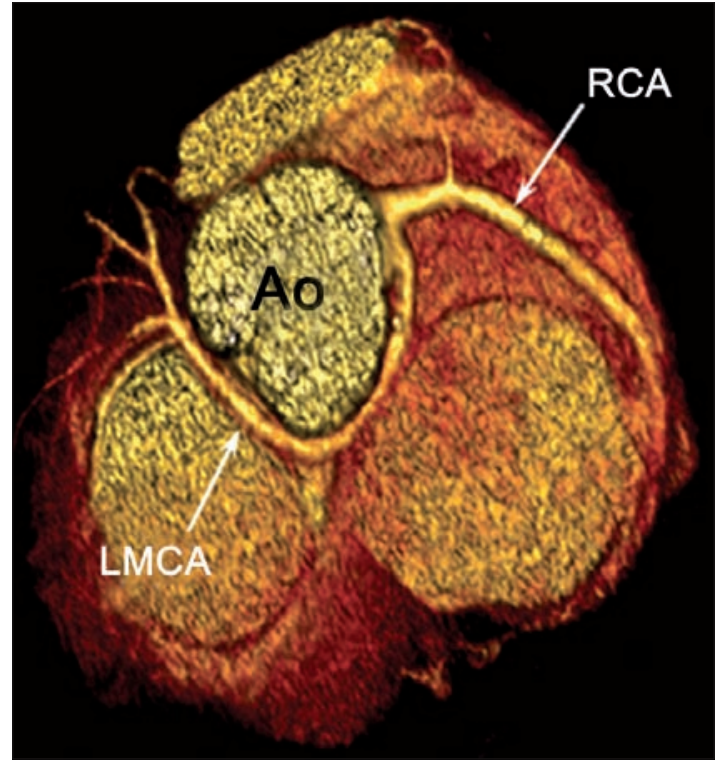

Figure 3. The left main coronary artery (LMCA), originating from the right sinus of Valsalva in a 37-year-old man. A volume-rendered display from a superior view demonstrates that the LMCA originates from the right sinus of Valsalva, and there is no coronary artery arising from the left sinus of Valsalva. Ao, aorta; RCA, right coronary artery. and follow either a septal course or an anterior free wall course; or d) the LADA may arise from the non-coronary aortic sinus, with its initial portion posterior to the aorta, and follow an anterior course toward its normal position (16).

Differentiation of this anomaly from a very short LMCA is difficult (17). Both anomalies can result in opacification of only the LADA or the left Cx artery on the CCA, which leads to the incorrect conclusion that one of the coronary arteries is completely occluded. However, with MDCT-CA, such an erroneous conclusion is avoided because the technique provides clear visualization of the proximal portion of the coronaries. Preoperative knowledge of this anomaly is crucial because the failure to perfuse one of the orifices during surgery could result in myocardial infarction (18).

\section{Ectopic origin of the LMCA from the right sinus of Valsalva}

An LMCA originating from the right sinus of Valsalva is a rare coronary anomaly $(6,19)$. An LMCA originating from the right sinus of Valsalva follows one of four courses: a septal, anterior free wall (Fig. 3), retroaortic, or interarterial course. Determining the course of the vessel is important because the interarterial course is frequently associated with a poor prognosis $(6,16$, 20). Unlike CCA, the MDCT-CA images can be reconstructed in multiple planes and in 3D; thus, MDCT-CA provides information on the route of the coronary arteries relative to the cardiac chambers and great vessels, ensuring an accurate diagnosis.

\section{Aorto-ostial lesions}

Aorto-ostial lesions are among the most important types of coronary artery disease, and these lesions occur within the first $3 \mathrm{~mm}$ of the main coronary vessel as the vessel splits from the aorta. Because complications are associated with aorto-ostial lesions, proper diagnosis and treatment are crucial $(1,21)$.

In a study involving 33 patients, Kantarci et al. (1) showed that MDCTCA diagnosed aorto-ostial lesions correctly in 26 patients. The remaining five patients with normal ostia were also correctly identified with MDCTCA. Seven out of 26 patients with aorto-ostial lesions were not detected with CCA. The authors postulated that this lack of detection likely resulted from positioning the catheter tip beyond the lesion (Fig. 4a and 4b). In addition, five patients with normal ostia were reported to have aorto-ostial lesions using CCA. Catheter-induced spasm is a significant complication associated with CCA. Although the spasm can be resolved by nitroglycerine infusion, it can sometimes be erroneously diagnosed as coronary stenosis. In the study by Kantarci et al. (1), this false diagnosis occurred in five patients whose coronary arteries were found to be normal using MDCT (Fig. 4c and 4d). Therefore, MDCT-CA is useful in preventing false diagnosis due to catheterinduced spasm in patients who have been diagnosed with an aorto-ostial lesion by CCA.

\section{Follow-up of bypass grafts}

Coronary artery bypass graft (CABG) surgery has become the mainstay treatment of symptomatic multiple coronary artery disease. Graft patency is the most important variable determining the effectiveness of the CABG surgery $(22,23)$. CCA has been considered the gold standard technique for assessing CABGs; however, it is invasive, requires hospitalization, and carries the risk of complications. Moreover, catheterization of the CABG may not always be possible $(21,24-26)$. In addition, in patients with multiple CABGs arising consecutively from the aorta, it may be difficult to determine which one is cannulated using CCA (Fig. 5). To evaluate the patency of the CABGs, distal graft anastomosis, and run-off vessels, MDCT-CA can be used (27). 

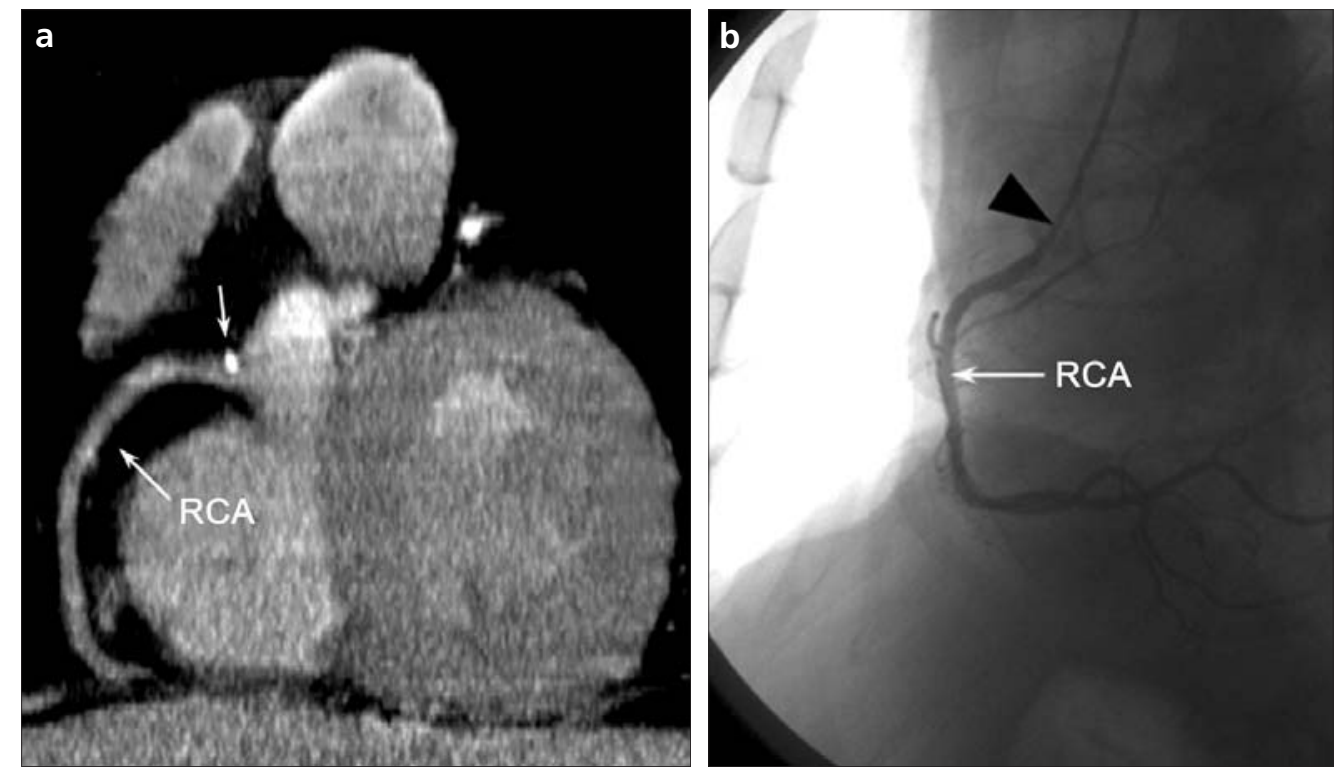

Figure 4. a-d. A 43-year-old man with an aorto-ostial lesion at the right coronary artery (RCA) $(\mathbf{a}, \mathbf{b})$. The sagittal MIP image from MDCT of the heart shows a calcified, discrete plaque (arrow) in the ostium of the RCA (a). Catheter angiography fails to show ostial stenosis because of the positioning of the catheter tip beyond the lesion (black arrowhead, catheter) (b). A 64-year-old woman with a normal ostium (c, d). A catheter angiogram shows decreased vessel caliber that is consistent with severe stenosis at the ostium of the left main coronary artery (LMCA) (arrowhead, catheter) (c, black arrow). In the same patient, an axial MIP image from MDCTCA shows the normal LMCA ostium (d). LADA, left anterior
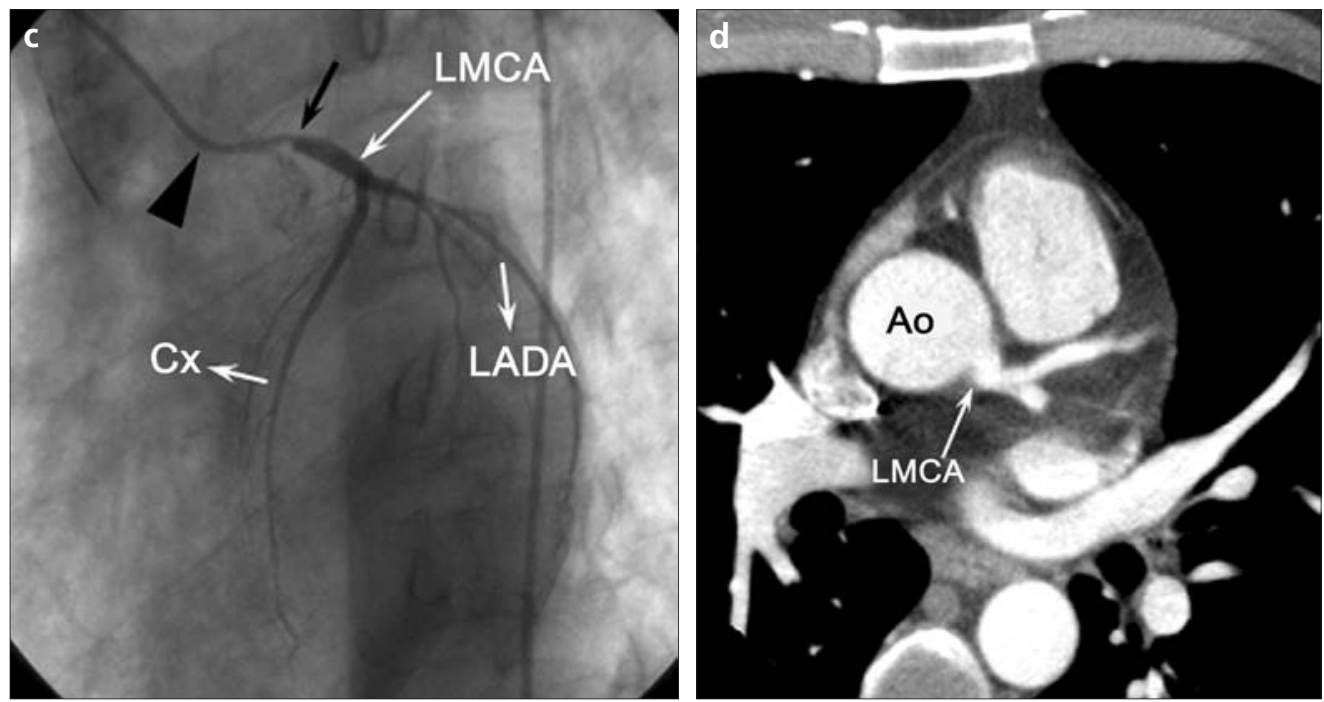
descending artery; Ao, aorta; $C x$, circumflex artery.

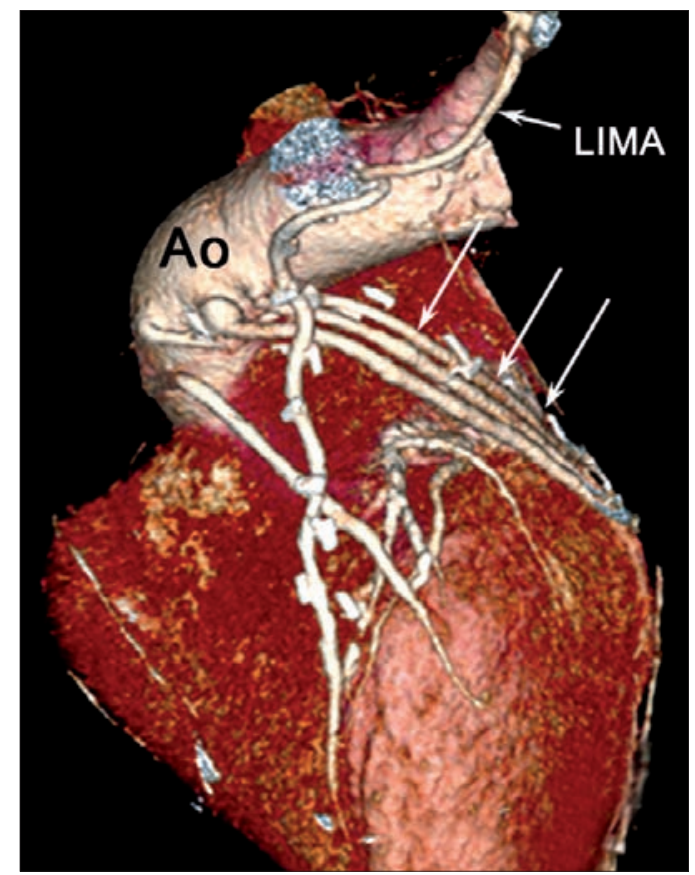

Figure 5. A 77-year-old man with multiple bypass grafts. Three consecutive in situ grafts (arrows) extend from the aorta to the circumflex and the obtuse marginal arteries. Also note the left internal mammary artery (LIMA)-left anterior descending artery graft. Ao, aorta.

\section{Myocardial bridging}

Myocardial bridging is a congenital anomaly characterized by myocardial encasement of a coronary artery segment that normally has an epicardial course. It is also called a tunneled artery (28). Myocardial bridging may cause clinical symptoms including angina, myocardial infarction, life-threatening arrhythmias, and sudden death $(28,29)$. Ferreira et al. (30) categorized myocardial bridging as either superficial ( $75 \%$ of cases) or deep (25\% of cases). Normally, the majority of myocardial blood flow is in diastole, and thus, systolic compression of the tunneled segment (i.e., superficial bridging) alone cannot sufficiently explain ischemia and its associated symptoms. However, deep myocardial bridging could compromise coronary diastolic 

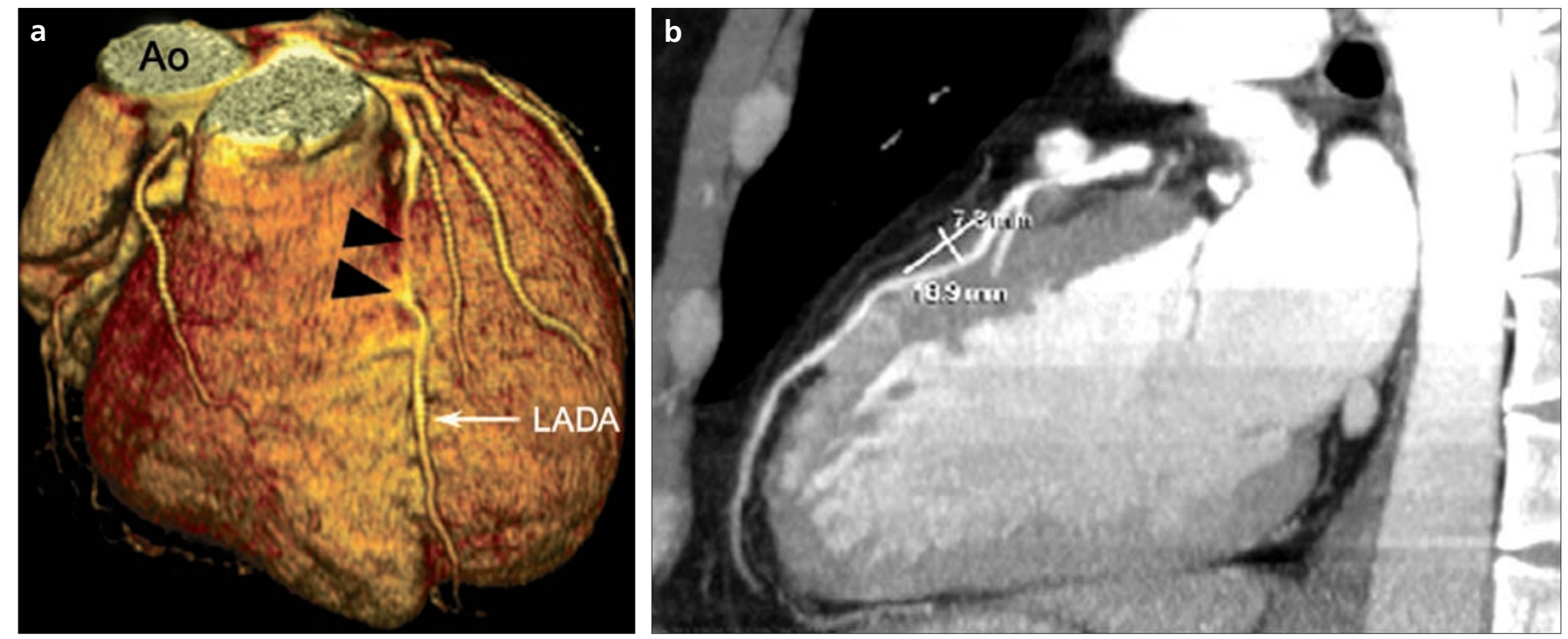

Figure 6. a, b. A 40-year-old man with myocardial bridging. A volume-rendered 3D image (a) shows myocardial bridging at the middle third of the left anterior descending artery (LADA) (arrowheads). At this tunneled segment, the caliber of the LADA appears thinner. The sagittal multiplanar reconstruction image (b) shows the intramyocardial course shifting into the myocardium of the middle LADA. The length and depth of the tunneled segment can be measured clearly using this image. Ao, aorta.
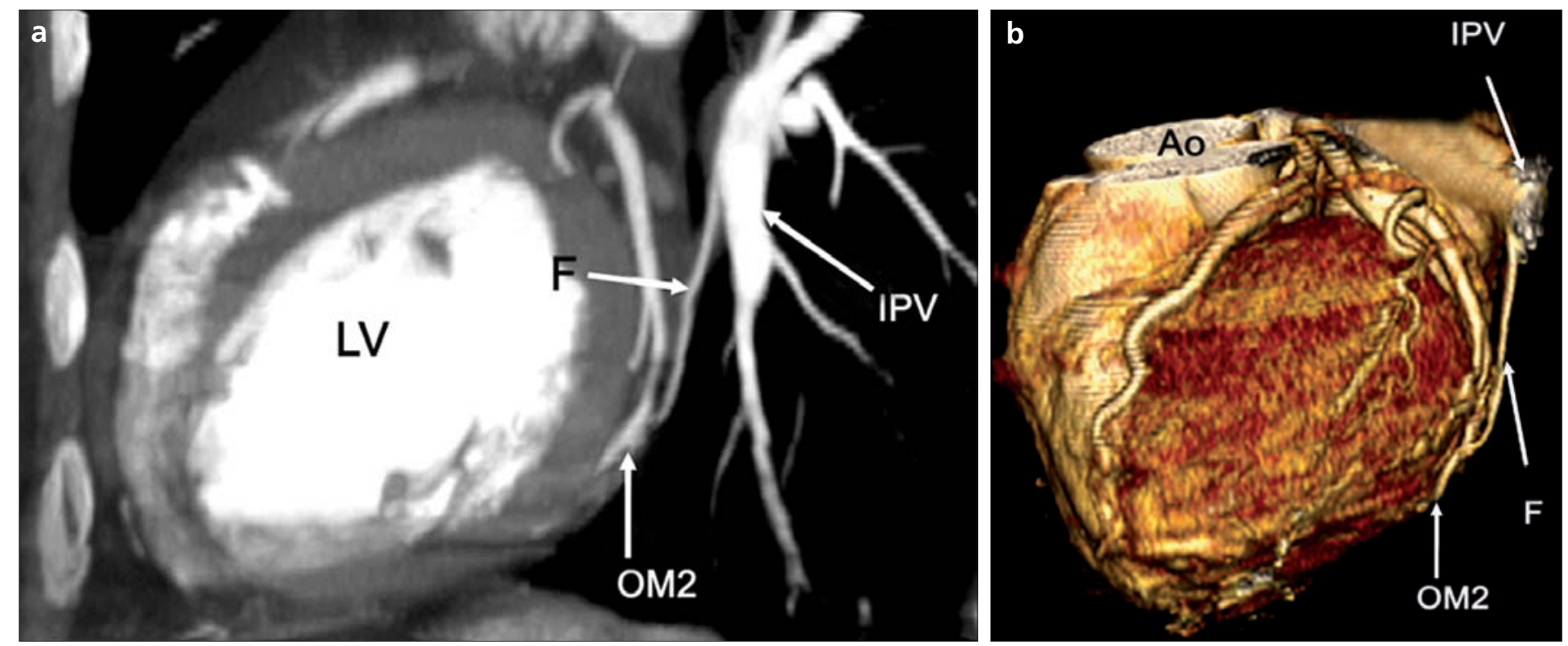

Figure 7. a, b. A 64-year-old woman with a coronary artery-pulmonary vein fistula. A sagittal MIP (a) and an anterior view, 3D volumerendered image (b) show a fistula between the obtuse marginal 2 (OM2, the second branch of the circumflex artery) and the left inferior pulmonary vein (IPV). F, fistula; LV, left ventricle; Ao, aorta.

flow and result in ischemia compared with superficial bridging (30-32).

The diagnosis of bridging with CCA can be challenging because the interpretation of catheter angiography findings requires an experienced eye, and only deep bridges can be detected with CCA $(29,33,34)$.

MDCT-CA can readily demonstrate an abnormal intramural course of the coronary artery through the myocardium, its depth, and the length of the involved segment can be readily demonstrated by MDCT-CA (Fig. 6) (29).
Dynamic evaluations can allow the evaluation of the degree of vascular stenosis during different phases of the cardiac cycle.

\section{Coronary artery fistulas}

Coronary artery fistulas are rare congenital or acquired coronary artery anomalies that can originate from any of the three major coronary arteries and that may drain into any cardiac chamber or great vessel (35). According to recent reports, coronary artery fistulas can be correctly identified using
MDCT (36-38). The use of MDCT-CA is an accurate, fast, robust, and non-invasive method of imaging for diagnosing coronary artery fistulas. Because CCA can identify only a single vessel during a single session, depending on the artery cannulated, in complicated conditions such as when a fistula is present, it may not be possible to determine exactly where the fistula drains (Fig. 7). In such cases, particularly those in which interventional procedures are scheduled, MDCT-CA provides more diagnostic information than CCA. 


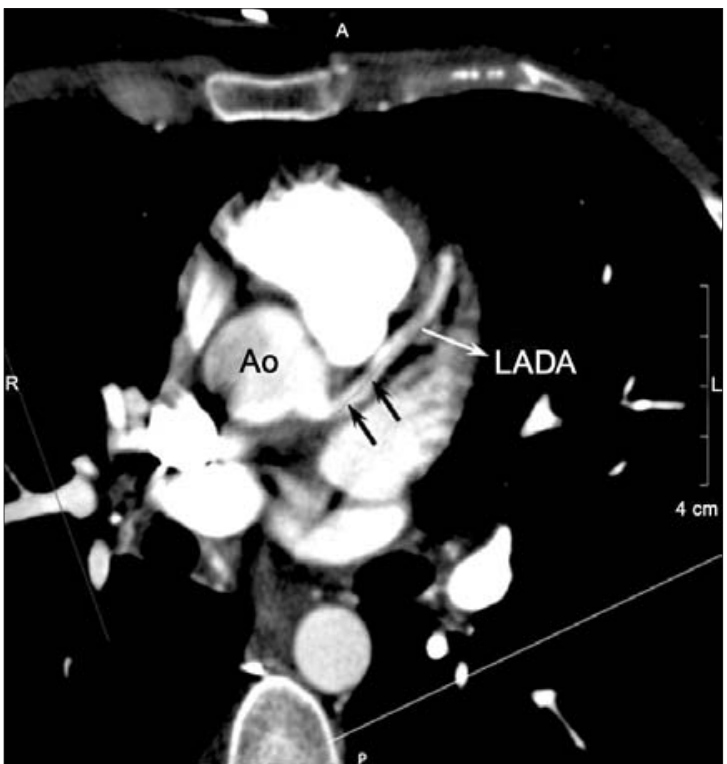

Figure 8. A 30-year-old woman with a coronary artery dissection. An axial contrast-enhanced MIP image from MDCT-CA shows a double lumen separated by an intimal flap (arrows) and a coronary dissection involving the proximal segment of the left anterior descending artery (LADA). Ao, aorta.

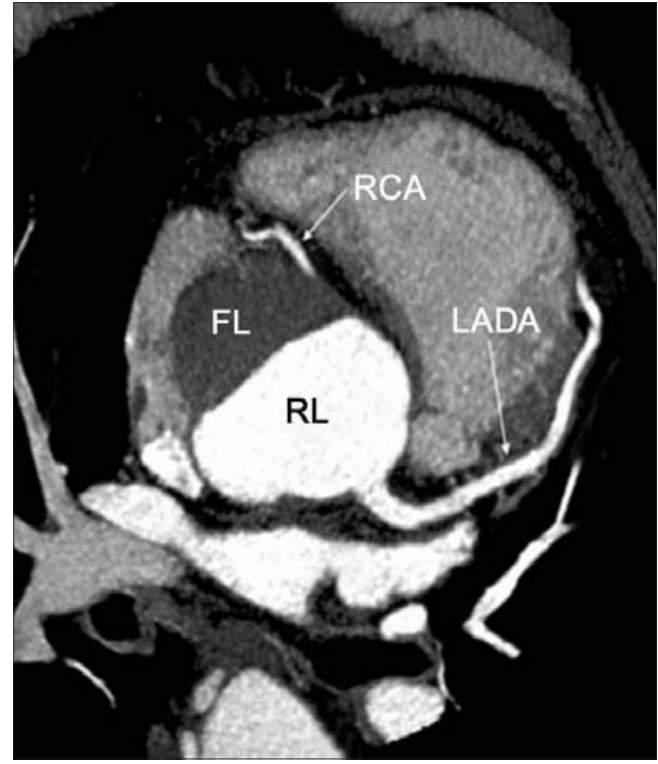

Figure 9. A 65-year-old man with a type 1 aortic dissection. In an axial MIP image provided by MDCT-CA, the aortic root, thrombosed false lumen (FL), and real lumen (RL) can be visualized. Coronary artery contrast enhancement is observed in both coronary arteries. RCA, right coronary artery.

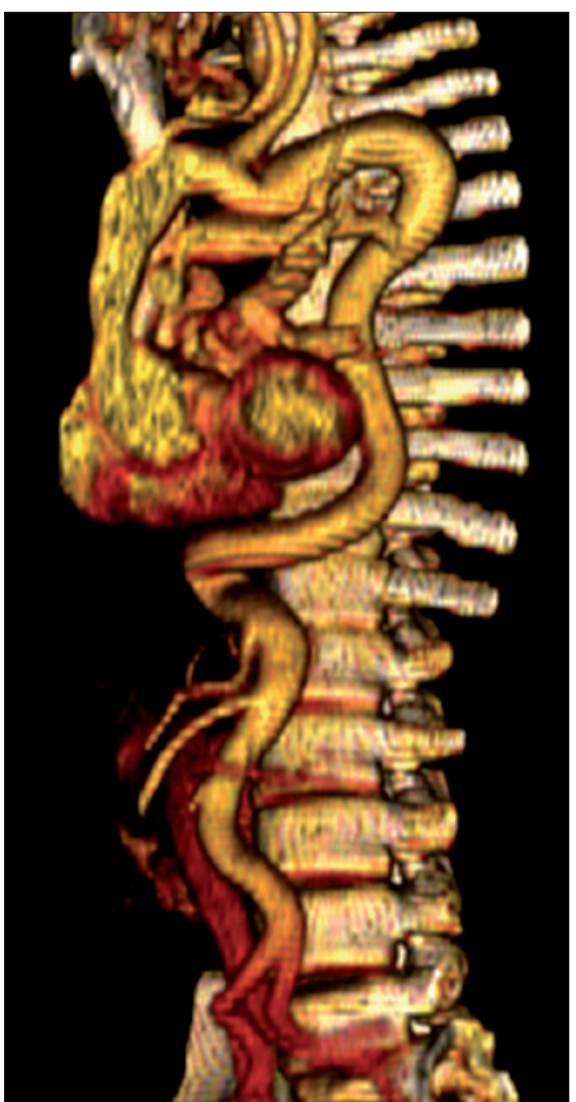

Figure 10. A 33-year-old man with extremely tortuous major vascular structures. A $3 \mathrm{D}$ volume-rendered reconstruction of the aorta from the anterior view demonstrates tortuosity and kinking in the abdominal aorta.

\section{Coronary artery dissection}

Dissection is a pathologic event that develops when blood dissects into the media layer through an intimal tear. When it affects the coronary arteries, it may cause life-threatening myocardial infarction, and therefore, it requires urgent diagnosis and treatment. Spontaneous coronary artery dissection predominantly affects young women, and while very rare, its effects are often severe (39). Spontaneous coronary artery dissection can lead to acute coronary syndrome or ischemic coronary events (40-42).

The size of the dissection may increase during CCA if the false lumen is inadvertently cannulated. Detecting whether the dissected segment extends into the coronary artery ostium is also important. In these cases, arrhythmia and myocardial infarct are common (40-42). The use of MDCT-CA allows noninvasive visualization of the presence and extension of a dissected segment as effectively as CCA (Fig. 8) (40-42).

Coronary arteries may also be secondarily involved in patients with aortic dissections. Dissection can involve the different parts of the aorta, and when it involves the ascending aorta, there is a risk for extension into the coronary arteries. A timely diagnosis and appropriate treatment is crucial because it is a life-threatening condition (40-43). During CCA, the catheter may pass through either the false or real lumen to reach the coronary ostia. Regardless of the main coronary artery ostium that is cannulated, it is difficult to cannulate the opposite ostium because of the intervening flap. Noninvasively, MDCT-CA demonstrates aortic root as well as coronary involvement at the same (Fig. 9). Any extension of the dissection into the coronary arteries and aortic ostium, the knowledge of which is important for preoperative planning, can also be demonstrated with MDCT-CA.

\section{Conditions in which access to coronary ostia is challenging}

Arterial tortuosity syndrome is a rare, autosomal recessive disorder of the connective tissue that is characterized by elongation, tortuosity, and aneurysms of the large- and medium-sized arteries (44). Tortuosity of the main vascular structures, caused by different vascular diseases, emerges as an important problem when using CCA imaging in patients with symptoms of angina who require catheterization. Tortuosity at the abdominal aorta and the axillary artery prevent the catheter from being advanced (Fig. 10); therefore, the catheter cannot reach the ostium. The most frequent cause of aortic aneurysm 

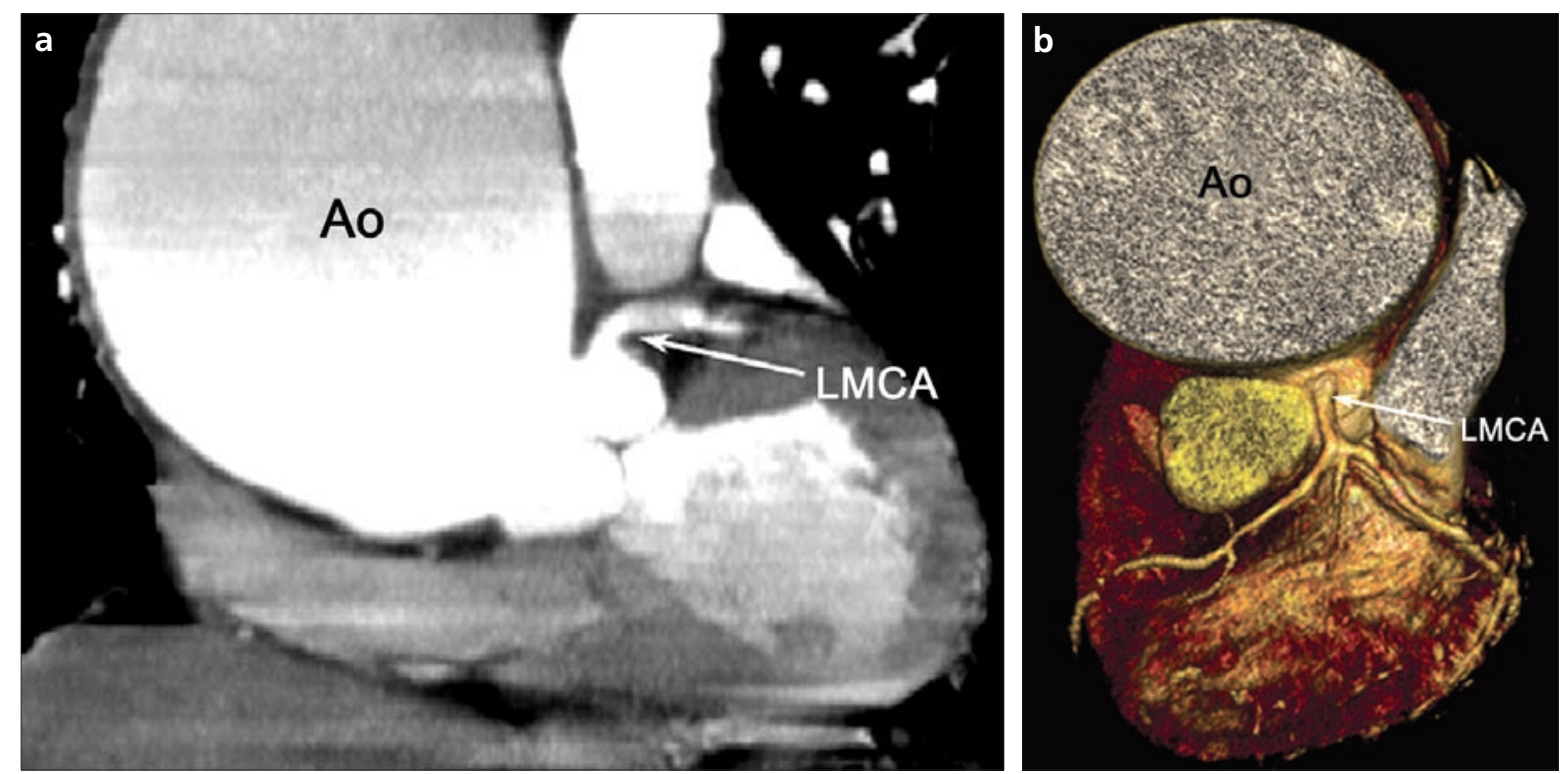

Figure 11. a, b. A 74-year-old man with a very large aortic aneurysm. Because of turbulent flow associated with catheter angiography, cannulation of the ostia was very difficult. The sagittal, contrast-enhanced MIP image from MDCT-CA (a) and a 3D volume-rendered reconstruction of the heart from a superior view (b) reveal an enormous ascending aortic aneurysm close to the main coronary arteries. Ao, aorta; LMCA, left main coronary artery.

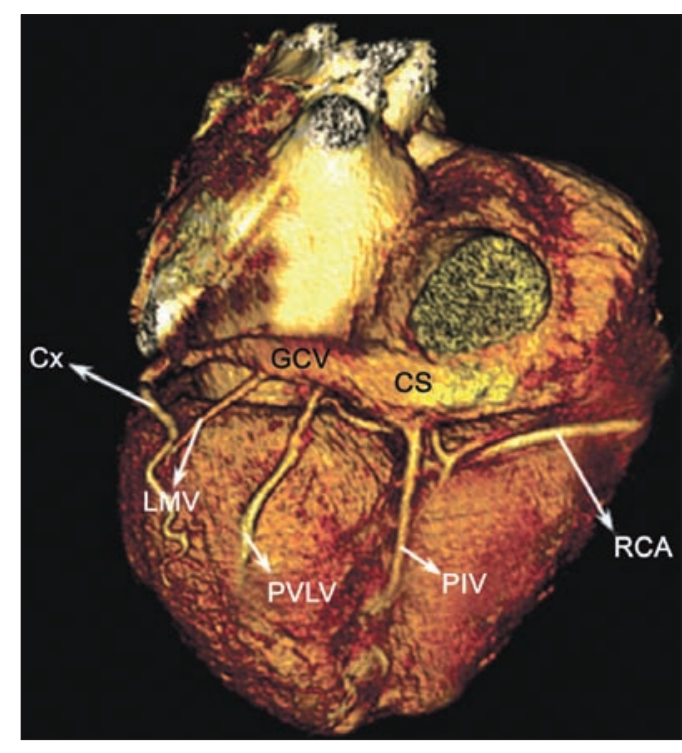

Figure 12. A 3D volume-rendered reconstruction of the heart from a posterior view in a 40 -year-old man with chest pain. The first tributary of the coronary sinus (CS) is the posterior interventricular vein (PIV), which runs in the posterior interventricular groove. The second tributary of the CS is the posterior vein of the left ventricle (PVLV). The third tributary is the left marginal vein (LMV). The great cardiac vein (GCV) then continues as the anterior cardiac vein in the anterior interventricular groove. Additionally, note the right coronary artery (RCA) and the circumflex (CX) artery. is atherosclerosis (45). Patients with massive aortic aneurysms encompassing the coronary artery ostia can be evaluated with MDCT-CA (Fig. 11) (46). Performing catheter angiography in these patients is very difficult because the widened lumen makes it almost impossible to locate the ostium with the catheter. In addition, turbulence in this region can adversely affect catheter control. In such patients, MDCT is the preferred approach for vessel visualization.

\section{Biventricular cardiac pacing}

Cardiac resynchronization therapy is a well-established treatment method for patients with heart failure (47). Implantation with cardiac resynchronization therapy will be successful only if the left ventricle lead can be positioned in a vein that drains this region. The left marginal vein and the posterolateral vein are often the target veins involved in pacemaker lead placement in cardiac resynchronization therapy $(48,49)$.

Biventricular cardiac pacing is a high-cost treatment method. It is important that the cardiologist have preprocedural information about the veins (Fig. 12). MDCT-CA can provide anatomic details of the cardiac venous anatomy in a noninvasive fashion and provide information regarding the size, number, location, and anatomic variants of the coronary veins so that appropriate catheters can be selected and ordered before performing the procedure (50).

\section{The presence of mediastinal tumors extending into the coronary arteries}

The heart is a target organ for many malignant tumors (51). Cardiac metastases are 20-40 times more common than primary cardiac malignancies. The malignancies that are most likely to spread to the heart are lung, breast, and esophageal cancers, as well as leukemia, lymphoma, and melanoma (Fig. 13) (52). If a patient with a primary or secondary cardiac malignancy presents with an angina requiring coronary angiography, the detection of the cause of angina is very important for selecting the appropriate treatment. Unlike CCA, which provides information only about the coronary artery lumen, MDCT-CA yields information about the heart, lungs, and other mediastinal and intrathoracic structures in addition to the coronary arteries (3).

\section{Possible drawbacks of MDCT-CA}

The major concerns about MDCTCA are related to radiation exposure and the use of injected contrast material (53). However, these concerns have become less serious since recent 


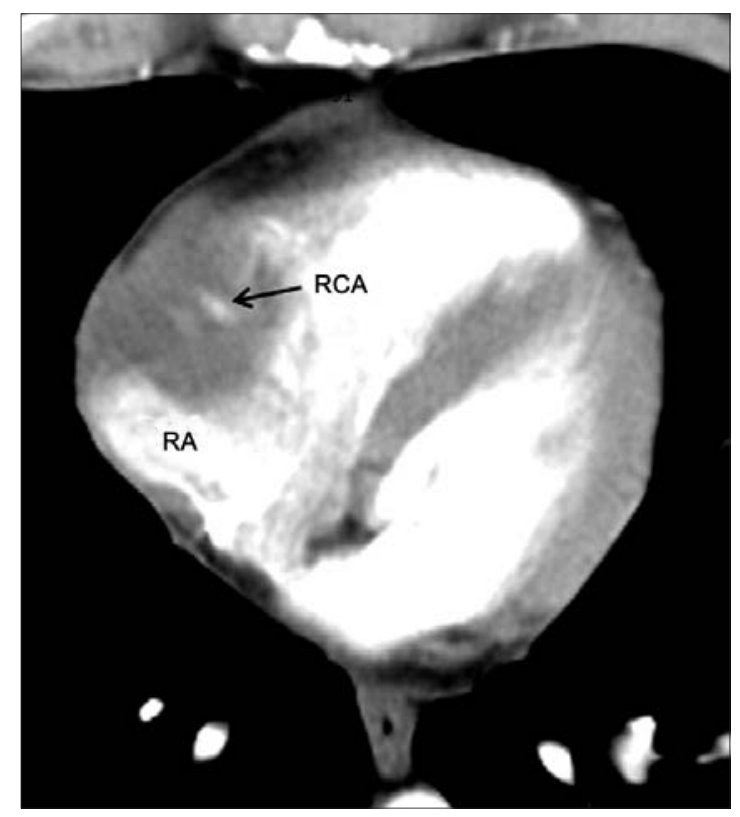

Figure 13. A 43-year-old man with a history of multiple lymphoma relapses who was referred with chest pain. An axial contrastenhanced CT shows infiltrative lymphoma encasing the right coronary artery (RCA) and invading the right atrium (RA).

advancements have dramatically reduced radiation dose and the iodinated contrast material requirement. Another disadvantage of MDCT-CA is the necessity of premedication to decrease heart rate. Despite the decreased required scanning time due to a new generation of equipment, breath holding still poses a problem in MDCT-CA, particularly in elderly patients with poor cardiopulmonary function. To some extent, these disadvantages could be overcome by using new-generation scanners that have rapid image acquisition, such as dual-source CT or scanners with 256 or more detectors (54).

As a conclusion, MDCT-CA has emerged as an accurate, fast, robust, and non-invasive tool for the comprehensive evaluation of the coronary vessels and heart chambers. The advantages of providing a volumetric dataset for the heart with an isotropic submillimeter resolution makes it a good fit for the assessment of congenital anomalies or acquired disease processes involving coronary vessels, heart chambers, and mediastinal structures. The use of MDCT-CA should substitute catheter angiography as the imaging modality of choice for a large spectrum of coronary disorders.

\section{Conflict of interest disclosure}

The authors declared no conflicts of interest.

\section{References}

1. Kantarci M, Ceviz N, Sevimli S, et al. Diagnostic performance of multidetector computed tomography for detecting aorto-ostial lesions compared with catheter coronary angiography: multidetector computed tomography coronary angiography is superior to catheter angiography in detecting of aorto-ostial lesions. J Comput Assist Tomogr 2007; 31:595-599.

2. Hoffman HK, Shi H, Schmid FT, Gelman $\mathrm{H}$, Brambs HJ, Aschoff AJ. Noninvasive coronary imaging with MDCT in comparison to invasive conventional coronary angiography. Am J Roentgenol 2004; 182:601-618.

3. Schroeder S, Achenbach S, Bengel F, et al. Cardiac computed tomography: indications, applications, limitations, and training requirements: report of a Writing Group deployed by the Working Group Nuclear Cardiology And Cardiac CT of the European Society of Cardiology and the European Council of Nuclear Cardiology. Eur Heart J 2008; 29:531-556.

4. Schoepf UJ, Becker CR, Ohnesorge BM, Yucel EK. CT of coronary artery disease. Radiology 2004; 232:18-37.

5. Leschka S, Stolzmann P, Desbiolles L, et al. Diagnostic accuracy of high-pitch dualsource CT for the assessment of coronary stenoses: first experience. Eur Radiol 2009; 19:2896-2903.

6. Duran C, Kantarci M, Subasi ID, et al. Remarkable anatomic anomalies of coronary arteries and their clinical importance: a multidetector computed tomography angiographic study. J Comput Assist Tomogr 2006; 30:939-948.

7. Kantarci M, Koplay M, Bayraktutan U, Gundogdu F, Ceviz N. Congenitally corrected transposition of the great atreries: MDCT angiography findings and interpretation of complex coronary anatomy. Int $\mathrm{J}$ Cardiovasc Imaging 2007; 23:405-410.
8. Sasaki O, Hamada M, Hiasa G, et al. Congenitally corrected transposition of the great arteries in a 65-year-old woman. Jpn Heart J 2001; 42:645-649.

9. Reddy GP, Caputo GR. Case 15: congenitally corrected transposition of the great arteries. Radiology 1999; 213:102-106.

10. Yilmaz-Cankaya B, Kantarci M, Yalcin A, Durur-Karakaya A, Yuce I. Absence of the left main coronary artery: MDCT coronary angiographic imaging. Eurasian J Med 2009; 41:56-58.

11. Lipton MJ, Barry WH, Obrez I, Silverman JF, Wexler L. Isolated single coronary artery: diagnosis, angiographic classification, and clinical significance. Radiology 1979; 130:39-47.

12. Kaku B, Shimizu M, Yoshio $\mathrm{H}$, et al. Clinical features of prognosis of Japanese patients with anomalous origin of the coronary artery. Jpn Circ J 1996; 60:731-741.

13. Kardos A, Babai L, Rudas L, et al. Epidemiology of congenital coronary artery anomalies: a coronary arteriography study on a central european population. Catheter Cardiovasc Diagn 1997; 42:270-275.

14. Garg N, Tewari S, Kapoor A, Gupta DK, Sinha N. Primary congenital anomalies of the coronary arteries: a coronary arteriographic study. Int J Cardiol 2000; 74:3946.

15. Serota H, Barth CW III, Seuc CA, Vandormael M, Aguirre F, Kern MJ. Rapid identification of the course of anomalous coronary arteries in adults: the "dot and eye" method. Am J Cardiol 1995; 65:891898.

16. Topaz O, De Marchena EJ, Perin E, Sommer LS, Mallon SM, Chahine RA. Anomalous coronary arteries: angiographic findings in 80 patients. Int J Cardiol 1992; 34:129 138.

17. Yilmaz-Cankaya B, Kantarci M, Yalcin A, Durur-Karakaya A, Yuce I. Absence of the left main coronary artery: MDCT coronary angiographic imaging. Eurasian J Med 2009; 41:56-58.

18. Cheitlin MD. Coronary arterial anomalies. In: Parmley WW, Chatterjee K, eds. Cardiology. Philadelphia: Lippincott, 1989; 9-11.

19. Abouzied AM, Amaram S, Neerukonda SK. Anomalous left coronary artery arising from right sinus of valsalva could be a minor congenital anomaly: a case report and review of the literature. Angiology 1999; 50:175-178.

20. Liberthson RR, Dinsmore RE, Fallon JT. Aberrant coronary artery origin from the aorta. Report of 18 patients, review of literature and delineation of natural history and management. Circulation 1979; 59:748-754.

21. Braundwald E. Coronary angiography and intravascular ultrasound imaging. In: Braunwald E, ed. Heart disease: a textbook of cardiovascular medicine, 6th ed. Philadelphia: Saunders, 2001; 496-497.

22. Hazirolan T, Turkbey B, Karcaaltincaba $\mathrm{M}$, et al. Impact of scanning direction on heart rate at certain levels of heart in electrocardiogram-gated 16-multidetector computed tomography angiography of coronary artery bypass grafts. J Comput Assist Tomogr 2007; 31:5-8. 
23. Frazier AA, Qureshi F, Read KM, Gilkeson RC, Poston RS, White CS. Coronary artery bypass grafts: assessment with multidetector CT in the early and late postoperative settings. Radiographics 2005; 25:881-896.

24. Johnson LW, Krone R. Cardiac catheterization 1991: a report of the registry of the society for cardiac angiography and interventions. Cathet Cardiovasc Diagn 1993; 28:219-220.

25. Durur-Subasi, Kantarci M, Durur-Karakaya A, Okur A. Comparison of 16-slice computed tomography with conventional angiography to evaluate coronary artery stent patency. Eurasian J Med 2009; 41:4-9.

26. Türkvatan A, Biyikoğlu SF, Büyükbayraktar FG, et al. Noninvasive evaluation of coronary artery bypass grafts and native coronary arteries: is 16-slice multidetector CT useful? Diagn Interv Radiol 2009;15:43-50.

27. Goetti R, Leschka S, Baumüller S, et al. Low dose high-pitch spiral acquisition 128-slice dual-source computed tomography for the evaluation of coronary artery bypass graft patency. Invest Radiol 2010; 45:324-330.

28. Hazirolan T, Canyigit M, Karcaaltincaba $\mathrm{M}$, et al. Myocardial bridging on MDCT. Am J Roentgenol 2007; 188:1074-1080.

29. Kantarci M, Duran C, Durur I, et al. Detection of myocardial bridging with ECG-gated MDCT and multiplanar reconstruction. Am J Roentgenol 2006; 186:391394.

30. Ferreira Jr AG, Trotter SE, Konig Jr B, Décourt LV, Fox K, Olsen EG. Myocardial bridges: morphological and functional aspects. Br Heart J 1991; 66:364-367.

31. Canyigit M, Hazirolan T, Karcaaltincaba $\mathrm{M}$, et al. Myocardial bridging as evaluated by 16 row MDCT. Eur J Radiol 2009; 69:156-164.

32. Berry JF, von Mering GO, Schmalfuss C, Hill JA, Kerensky RA. Systolic compression of the left anterior descending coronary artery: a case series, review of literature, and therapeutic options including stenting. Catheter Cardiovasc Interv 2002; 56:58-63.

33. Alegria JR, Herrmann J, Holmes DR Jr, Lerman A, Rihal CS. Myocardial bridging. Eur Heart J 2005; 26:1159-1168.

34. Dominguez B, Valderrama V, Arrocha $\mathrm{R}$, Lombana $\mathrm{B}$. Myocardial bridging as a cause of coronary insufficiency. Rev Med Panama 1992; 17:28-35.
35. Doganay S, Bozkurt M, Kantarci M, Erkut B. Coronary artery-pulmonary vein fistula diagnosed by multidetector computed tomography. J Cardiovasc Med 2009; $10: 428-430$.

36. Seguchi O, Terashima M, Awano K Multiple coronary artery fistulas visualised by three dimensional computed tomography. Heart 2003; 89:1381.

37. Gundogdu F, Arslan S, Buyukkaya E, Kantarci M. Coronary artery fistula in a patient with coronary artery disease: evaluation by coronary angiography and multidetector computed tomography. Int J Cardiovasc Imaging 2007; 23:299-302.

38. Dodd JD, Ferencik M, Liberthson RR, et al. Evaluation of efficacy of 64-slice multidetector computed tomography in patients with congenital coronary fistulas. J Comput Assist Tomogr 2008; 32:265-270.

39. Park SM, Koh KK, Kim JH, Yoon KH, Chung WJ, Kang WC. Myocardial infarction with huge mural thrombus due to spontaneous coronary artery dissection detected by 64-multidetector computed tomography. Int J Cardiol 2008; 127:73-75.

40. Kantarc1 M, Ogul H, Bayraktutan U, Gundogdu F, Bayram E. Spontaneous coronary artery dissection: noninvasive diagnosis with multidetector CT angiography. J Vasc Interv Radiol 2007; 18:687-688.

41. Roig S, GomezJA, Fiol M, et al. Spontaneous coronary artery dissection causing acute coronary syndrome: an early diagnosis implies a good prognosis. Am J Emerg Med 2003; 21:549-555.

42. Manghat NE, Morgan-Hughes GJ, Roobottom CA. Spontaneous coronary artery dissection: appearance and follow-up on multidetector row CT coronary angiography. Clin Radiol 2005; 60:1120-1125.

43. Murayama T, Funabashi N, Uehara $M$, Takaoka H, Komuro I. New classification of aortic dissection during the cardiac cycle as pulsating type and static type evaluated by electrocardiogram-gated multislice CT. Int J Cardiol 2010;142:177-186

44. Callewaert BL, Willaert A, KerstjensFrederikse WS, et al. Arterial tortuosity syndrome: clinical and molecular findings in 12 newly identified families. Hum Mutat 2008; 29:150-158.
45. Reardon MJ, Hedrick TD, Letsou GV, Safi HJ, Espada R, Baldwin JC. CT reconstruction of an unusual chronic posttraumatic aneurysm of the thoracic aorta. Ann Thorac Surg 1997; 64:1480-1482.

46. Ocak I, Lacomis M, Deible R, Türkbey B, Knollmann F. Prevalence of aortic root dilation in patients with CT angiography of the aorta. Diagn Interv Radiol 2011; $17: 272-276$

47. Mlynarski R, Sosnowski M, Wlodyka A, et al. A user- friendly method on cardiac venous system visualization in 64- slice computed tomography. Pace 2009; 32:323329.

48. Saremi F, Krishnan S. Cardiac conduction system: anatomic landmarks relevant to interventional electrophysiologic techniques demonstrated with 64-detector CT. Radiographics 2007; 27:1539-1567.

49. Van de Veire NR, Schuijf JD, De Sutter J, et al. Non-invasive visualization of the cardiac venous system in coronary artery disease patients using 64-slice computed tomography. J Am Coll Cardiol 2006; 48:1832-1838.

50. Hasdemir C. Cardiac resynchronization therapy: implantation tips and tricks. Anadolu Kardiyol Derg 2007; 7:53-56.

51. Dursun M, Sarvar S, Cekrezi B, Kaba E, Bakir B, Toker A. Cardiac metastasis from invasive thymoma via the superior vena cava: cardiac MRI findings. Cardiovasc Intervent Radiol 2008; 31:209-212.

52. Sosvinska-Mielcarek K, Senkus-Konefka E, Jassem J. Cardiac involvement at presentation of non-small-cell lung cancer. J Clin Oncol 2008; 26:1010-1011.

53. Vural M, Ucar O, Selvi A, et al. Assessment of global left ventricular systolic function with multidetector CT and 2D echocardiography: a comparison between reconstructions of $1-\mathrm{mm}$ and 2-mm slice thickness at multidetector CT. Diagn Interv Radiol 2010;16:236-240.

54. Karcaaltıncaba M, Aktas A. Dualenergy CT revisited with multidetector CT: review of principles and clinical applications. Diagn Interv Radiol 2011;17:181194. 